www.jmscr.igmpublication.org

Index Copernicus Value: 79.54

ISSN (e)-2347-176x ISSN (p) 2455-0450

crossrefDOI: https://dx.doi.org/10.18535/jmscr/v7i3.114

\title{
Cat Scratch Disease: Report of 2 cases
}

\author{
Authors \\ Dr Anurita Saigal, Dr Anchana Gulati, Dr Rajni Kaushik, Dr Neelam Sharma \\ Department of Pathology, Indira Gandhi Medical College, Shimla \\ Corresponding Author \\ Dr Anurita Saigal \\ Department of Pathology, Indira Gandhi Medical College, Shimla, India
}

\section{Introduction}

Cat scratch disease (CSD) also known as "Cat scratch fever" or "Subacute regional lymphadenitis" is usually a benign infectious disease caused by the intracellular bacterium Bartonella henselae ${ }^{1}$. It is a necrotizing granulomatous lymphadenitis most commonly found in children. It is a self limited infection caused by cutaneous inoculation of the bacteria introduced by a scratch, bite, licking of a cat. It was first discovered in 1889 by Henri Parinaud ${ }^{2}$. Kittens are more likely to carry the bacteria in their blood, and may therefore more likely to transmit the disease than adult cats. However, the results of experimental studies showed that fleas serve as a vector for transmission of B. henselae among cats ${ }^{3}$. It comes into clinical attention due to lymphadenopathy following infection. Regional lymphadenopathy appears after 1-3 weeks which is usually unilateral in $50 \%$ cases. Axillary, epitrochlear, cervical, or inguinal lymph nodes are commonly involved. One lymph node is involved in $50 \%$ of cases and multiple nodes, usually in the same region, in $30 \%$ of cases. In the general population, CSD is a benign, self-limited illness, lasting 6 to 12 weeks in the absence of treatment.
We are reporting two cases of 53 year old male and 10 year old female with cervical lymphadenopathy. Cytology was suggestive in the first case and excision biopsy was diagnostic in the second case. History of contact with cats supported the diagnosis.

\section{Case Report}

Case 1: A 53 year old male presented with right side cervical lymphadenopathy of 2 months duration. There was no history of fever/weight loss/cough. No history of ATT intake was present. On examination, the swelling was $0.8 \times 0.6 \mathrm{~cm}$ in size, non tender, mobile, firm and overlying skin was normal. FNAC was performed.

Microscopic Examination of FNA showed cellular smears revealing polymorphous lymphoid cell population comprising small mature lymphocytes, follicular center cells, immunoblasts. Plasma cells, neutrophils, histiocytes, epithelioid histiocytes, small epithelioid aggregates and multinucleate histiocytes were also seen along with focal purulent exudate/necrotic debris. (Figure 1). ZN Staining: No AFB was demonstrared. 
History of contact with cats was elicited. Keeping in view the clinical details and cytological findings, possibility of cat-scratch disease was suggested.

In this case azithromycin was given and patient followed up for 3 months. The swelling regressed and biopsy wasn't performed.

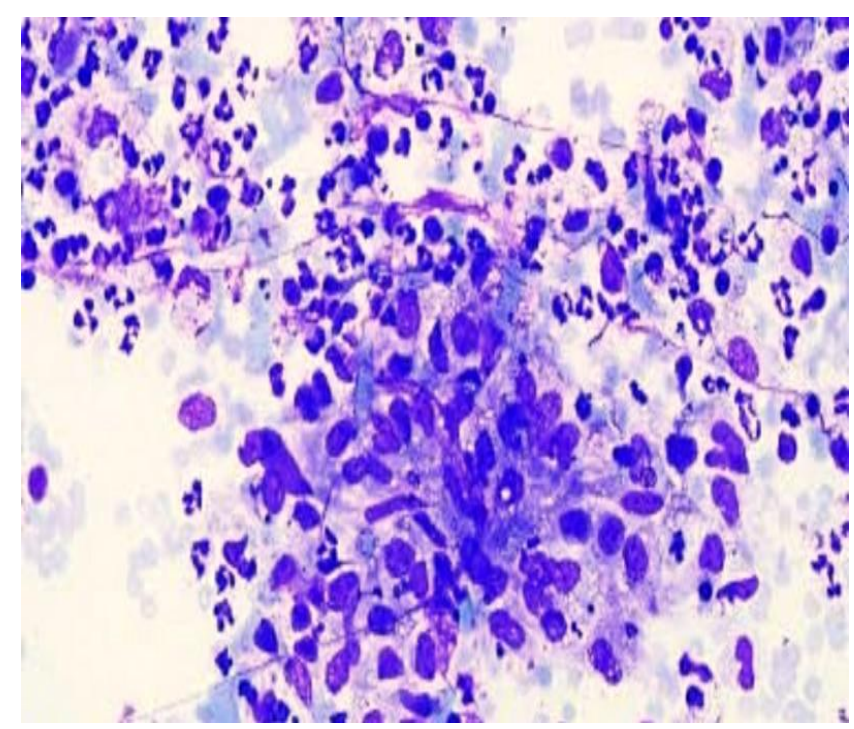

Figure 1: Epithelioid aggregates along with neutrophils

Case 2: 10 year old female presented with cervical lymphadenopathy of one month duration. There was no history of fever/weight loss/cough. There was history of intake of ATT in mother. On examination $0.5-1 \mathrm{~cm}$, mobile, non-tender cervical lymphnode. FNAC was performed and revealed cytologic features of non-specific reactive hyperplasia. Excision biopsy was done to rule out tuberculosis.

\section{Gross}

Received 2 lymph nodes measuring $1.5 \times 0.6 \times 0.2$ $\mathrm{cm}$ and $1.6 \mathrm{x} 0.3 \mathrm{x} 0.1 \mathrm{~cm}$ respectively.

\section{Histopathology}

Microscopic examination of lymph node revealed proliferation of lymphoid follicles of variable shape and size with prominent germinal centres and interspersed tingible body macrophages. (follicular hyperplasia). The interfollicular area also revealed many scattered macrophage. (Figure 2) A part of lymphnode revealed multiple small foci revealing aggregates of neutrophils admixed with lymphocytes and histiocytes. (Figure 3)

There was history of contact with cats and diagnosis of Cat Scratch disease was given

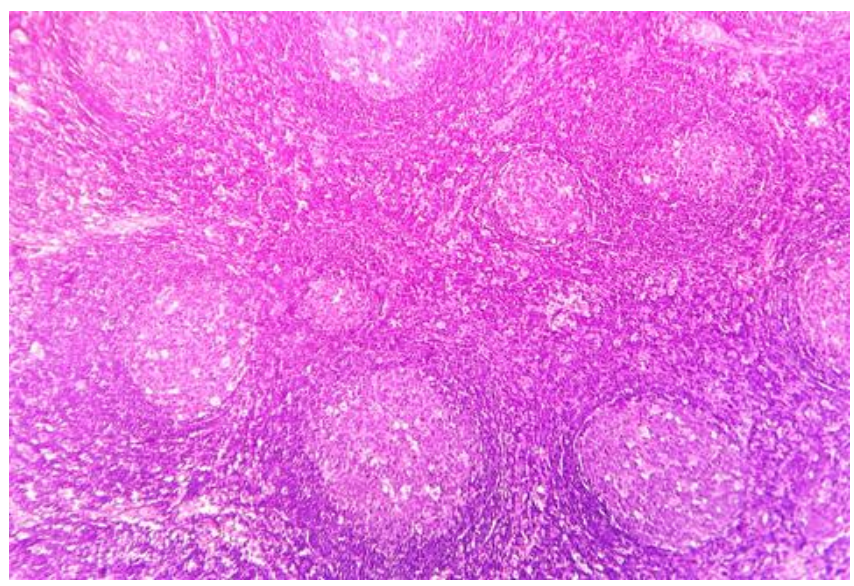

Figure 2: Follicular hyperplasia with interspersed tingible body macrophages

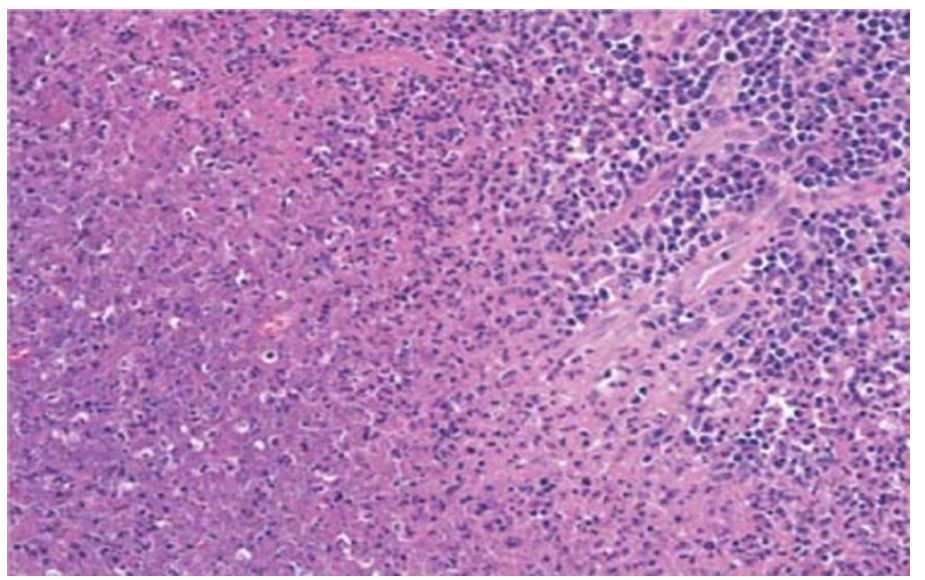

Figure 3: Central area of necrosis (left) surrounded by neutrophils (center), and lymphocytes (right)

\section{Discussion}

Cat sctrach disease is a less frequently reported disease entity. Chronic regional lymphadenopathy is the most common clinical feature of CSD and usually develops about 2 weeks after the scratch or contact with cat. The enlarged tender lymphnodes are most commonly found in the head or neck areas ${ }^{4}$. The axillary nodes are frequently involved, less commonly epitrochlear, inguinal, femoral, rarely supraclavicular nodes may be enlarged. Single node involvement occurs in 50\% of the patients. $20 \%$ involvement of multiple 
lymphnodes in the same site occurs. About one third of patients have lymphnode enlargement involving several sites. Node enlargement persists for 2-4 months, last for upto two years. Suppuration of involved lymphnode occurs in $10 \%$ of patients ${ }^{5}$.An inoculation site may be detected in $2 / 3^{\text {rd }}$ patients. Low grade fever $(30 \%)$, malaise $(20 \%)$, headache and sore throat (10\%) may be the presenting complaints.

CSD should be suspected if the patient has a history of exposure to cats and develops lymphadenopathy and a skin lesion. Fine needle aspiration cytology reveals characterstic granulomas with peripherally palisading epithelioid histiocytes and centrally located neutrophils and an associated polymorphous cell population. The diagnosis can be confirmed by pathological examination of the involved nodes. The histopathologic features of lymph nodes are consistent but not pathognomonic ${ }^{1}$. Early lymph node changes consist of follicular hyperplasia with preservation of the general architecture .Numerous tingible-body macrophage activity and the deposition of pink, amorphous intercellular proteinaceous material are characteristic. Vascular proliferation and patches of clear, monocytoid cells are also seen in this early stage.Small abscesses with focal necrosis and clusters of neutrophils appear first under the subcapsular sinus, progressing then from the cortex into the medulla .Sometimes, tiny bacilli in clusters can be seen in biopsy samples stained with Warthin Starry silver stain. The gold standard for diagnosing CSD is by an indirect immunofluorescent antibody assay or IFA ${ }^{4}$. If CSD is present, high titers of antibody will react to the B. henselae antigen. Titer of 1:64 or higher is considered positive. The identification of $\mathrm{B}$ Henselae 16 S ribosomal Ribo Nucleic Acid genes in biopsy material by Polymerase Chain Reaction Amplification with specific oligonucleotide primers can also be diagnostically useful, but these methods are not commercially available ${ }^{6}$.

\section{Conclusion}

Cat-Scratch Disease is a self limiting chronic regional lymphadenitis which can occur in those who have contact with cats. So it should be borne in mind as a differential diagnosis, while evaluating chronic lymphadenitis. Most patients with cervical lymphadenopathy need focused histopathological diagnosis. There may be varied clinical presentation of the same pathological entity. The history of "Cat Scratch" preceding the initial presentation to the doctor can help in diagnosis especially when all corroborative radiological and laboratory analysis for Tuberculosis is negative. Fortunately it is selflimited and no specific treatment is necessary.

\section{References}

1. Rapini, Ronald P, Bolognia, Jean L., Jorizzo, Joseph L. Dermatology: 2Volume Set. St. Louis: Mosby. ISBN 14160-2999-0

2. Jerris RC, Regnery RL (1996). "Will the real agent of cat-scratch disease please stand up?" Annu. Rev. Microbiol. 50: 707-25

3. Chomel BB, Kasten RW, Floyd-Hawkins $\mathrm{K}$, et al. "Experimental transmission of Bartonella henselae by the cat flea". J. Clin. Microbiol. August 1996. 34 (8): 1952-6.

4. Ioachim HL, Medeiros LJ. Ioachim's Lymph node pathology. 4. Philadelphia: Lippincott Williams \& Wilkins; 2009. CatScratch Lymphadenitis; pg. 110-114.

5. Mandell, Douglas and Bennett's Principles and practice of Infectious Diseases - IV Edition Volume II 108:1310 $-1312$

6. Manual of Clinical Microbiology by Patrick R Murray, \& Ellen Jo Baron, James H, Jorgensen, Michael A, Pealler, Robert, H Yolken 8th edition Vol 1, Pg 825-826. 